\title{
Contrast-enhanced computed tomography prior to percutaneous transthoracic needle biopsy reduces the incidence of hemorrhage
}

\author{
Huan $\mathrm{Hu}^{1 \#}$, Chuling $\mathrm{Li}^{2 \#}$, Tangfeng $\mathrm{Lv}^{3}$, Huijuan $\mathrm{Li}^{2}$, Yangbo $\mathrm{Hu}^{4}$, Qin $\mathrm{Shen}^{5}$, Mari Mino-Kenudson ${ }^{6}$, \\ Luca Bertolaccini $^{7}$, Gaetano Rocco ${ }^{8}$, Pavlos Zarogoulidis ${ }^{9}$, Fang Zhang $^{3}$, Dang Lin $^{1}$, Hongbing Liu ${ }^{3}$, \\ Yong Song ${ }^{2,3}$; written on behalf of AME Pulmonary Disease Collaborative Group
}

${ }^{1}$ Department of Respiratory Medicine, Suzhou Hospital Affiliated Nanjing Medical University, Suzhou, China; ${ }^{2}$ Department of Respiratory Medicine, Jinling Hospital, Nanjing Medical University, Nanjing, China; ${ }^{3}$ Department of Respiratory Medicine, Jinling Hospital, Nanjing University School of Medicine, Nanjing, China; ${ }^{4}$ Department of Respiratory Medicine, Jinling Hospital, Medical School of Southeast University, Nanjing, China; ' Department of Pathology, Jinling Hospital, Nanjing University School of Medicine, Nanjing, China; ${ }^{6}$ Department of Pathology, Massachusetts General Hospital, Boston, MA, USA; ${ }^{7}$ Division of Thoracic Surgery IEO, European Institute of Oncology IRCCS, Milan, Italy; ${ }^{8}$ Department of Thoracic Surgery and Oncology, National Cancer Institute, Pascale Foundation, Naples, Italy; ${ }^{9}$ Pulmonary Department-Oncology Unit, "G. Papanikolaou” General Hospital, Aristotle University of Thessaloniki, Thessaloniki, Greece

Contributions: (I) Conception and design: D Lin, H Liu, Y Song; (II) Administrative support: Q Shen, F Zhang, D Lin, Y Song; (III) Provision of study materials or patients: C Li, T Lv, H Liu; (IV) Collection and assembly of data: $\mathrm{H} \mathrm{Hu}, \mathrm{H} \mathrm{Li}, \mathrm{Y} \mathrm{Hu}$; (V) Data analysis and interpretation: C Li, M Mino-Kenudson, L Bertolaccini, G Rocco, P Zarogoulidis; (VI) Manuscript writing: All authors; (VII) Final approval of manuscript: All authors.

\#These authors contributed equally to this work.

Correspondence to: Dang Lin. Department of Respiratory Medicine, Suzhou Hospital Affiliated Nanjing Medical University, 16 West Baita Road, Suzhou 215001, China. Email: Danglin4067@163.com; Hongbing Liu. Department of Respiratory Medicine, Jinling Hospital, Nanjing University School of Medicine, 305 East Zhongshan Road, Nanjing 210002, China. Email: netlhb@126.com; Yong Song. Department of Respiratory Medicine, Jinling Hospital, Nanjing Medical University, 305 East Zhongshan Road, Nanjing 210002, China. Email: yongsong6310@yahoo.com.

Background: Hemorrhage is the second most common complication of percutaneous transthoracic needle biopsy (PTNB), and at present, there is no effective prevention strategy. Contrast-enhanced computed tomography (CECT) has the advantage of clearly visualizing blood supply within the lesion and aiding in the imaging of blood vessels, which can reduce hemorrhage complicating PTNB. As no large-sample studies were evaluating whether CECT could reduce hemorrhage, we conducted the present retrospective study.

Methods: From November 2011 to February 2016, 1,282 biopsies at Jinling Hospital were retrospectively reviewed; 555 underwent CECT, and 727 underwent non-contrast computed tomography (CT). Factors associated with hemorrhage were defined, and hemorrhage rates were compared between the 2 groups.

Results: We found that pre-biopsy CECT was associated with a reduced incidence of biopsy-related hemorrhage compared to non-contrast CT (16.4\% vs. 23.1\%, P=0.003). Propensity score matching (PSM) analysis also showed that the incidence of hemorrhage in the CECT group was lower than that of the noncontrast CT group at a ratio of $1: 1(\mathrm{P}=0.039), 1: 2(\mathrm{P}=0.028)$, or $1: 3(\mathrm{P}=0.013)$. In the multivariate analysis, CECT before PTNB was found to be significantly associated with a reduced risk of hemorrhage [odds ratio (OR): 0.671, 95\% confidence interval (CI): 0.499-0.902, $\mathrm{P}=0.008$ ]. Puncture position, lesion size, depth of needle tract, and the number of punctures were also found to be associated with hemorrhage (all $\mathrm{P}<0.05$ ).

Conclusions: Compared with non-contrast CT, CECT significantly reduced the risk of post-biopsy pulmonary hemorrhage, which suggests that CECT should be performed before PTNB.

Keywords: Percutaneous transthoracic needle biopsy (PTNB); contrast-enhanced computed tomography (CECT); hemorrhage

Submitted May 31, 2020. Accepted for publication Nov 06, 2020.

doi: 10.21037/atm-20-4384

View this article at: http://dx.doi.org/10.21037/atm-20-4384 


\section{Introduction}

Percutaneous transthoracic needle biopsy (PTNB) is a minimally invasive and safe procedure widely used for diagnosing lung lesions and the analysis of molecular alterations in clinical practice (1-5). Up to $2.9-54.5 \%$ of patients who undergo PTNB develop hemorrhage (3,6-10). Reported risk factors for pulmonary hemorrhage include penetration of blood vessels in the lung $(11,12)$. Although most pulmonary hemorrhage is mild and self-limiting, severe hemorrhage might also occur during PTNB $(8,13)$.

Contrast-enhanced computed tomography (CECT) has the advantage of clearly visualizing blood supply within the lesion and aiding in blood vessels' imaging. We, therefore, postulate that CECT could be used to reduce hemorrhage. The British Thoracic Society guidelines recommend performing computed tomography (CT) scan before lung biopsy (14), but do not mention whether or not CECT scans should be performed. Compared with non-contrast CT, CECT facilitates more accurate blood vessel imaging, particularly small vessels (15). Previous reports have indicated that most patients undergo CECT scans before the intervention to evaluate the feasibility of biopsy $(10,16)$, but no large cohort study evaluating the effect of pre-biopsy CECT on hemorrhage after a biopsy has been conducted. Therefore, we assessed the efficacy of pre-biopsy CECT in reducing PTNB-related hemorrhage. In the present study, we compared hemorrhage rates between the non-contrast $\mathrm{CT}$ and the CECT groups and identified the risk factors associated with pulmonary hemorrhage during the biopsy.

We present the following article following the STROBE reporting checklist (available at http://dx.doi.org/10.21037/ atm-20-4384).

\section{Methods}

Although the present study was a retrospective study, the propensity score matching (PSM) method matched confounding factors, making the results more reliable.

\section{Participants}

We retrospectively reviewed 1,430 biopsy procedures, which were performed on 1,370 patients between November 2011 and February 2016 in Jinling Hospital, Nanjing, China. To avoid the impact of repeat puncture targeting the same lesion on hemorrhage rates, for cases with repeated biopsies (49 patients), only the first biopsy was included in the analysis. Fifty patients with incomplete medical data were also excluded. Finally, a total of 1,271 patients (1,282 biopsies) were enrolled, including 11 patients with 2 independent lesions; the second biopsies were defined as independent incidences (Figure 1). The present study was conducted following the Declaration of Helsinki (as revised in 2013) and approved by the institutional review board of Jinling Hospital (No. 2017NZHX-022). All data were retrieved from a prospectively collected electronic database. Informed consent from individuals was waived due to the retrospective, observational, and anonymous nature of the current study.

\section{Inclusion criteria for biopsy}

Patients included in the present study had focal lung lesions appearing as a nodule or a mass. Laboratory indices relevant to the hemorrhage complication, such as prothrombin time, activated partial thromboplastin time, international normalized ratio, and platelet count within 1 week of undergoing PTNB, were normal. Patients with a medical history of anticoagulant or antiplatelet medication use were required to stop taking medication at least 5 days before puncture, and those patients have also included if re-examination of coagulation was normal. Those who could not follow verbal or visual instruction and/or accept procedure-related risks were excluded from the study.

\section{Biopsy procedure}

A helical CT scanner (SOMATOM Spirit; Siemens Medical Systems, Forchheim, Germany) and BARD MAGNUM 18-gauge cutting needles (Bard Peripheral Vascular, Inc., Tempe, Arizona, USA) were used. All patients underwent either chest CECT or non-contrast CT before the biopsy. Following a review of the pre-biopsy CT images, the optimal needle path was planned to perforate the least amount of aerated lung. Following sterilization and local anesthesia, a coaxial 18-gauge needle was inserted through the chest wall and moved towards the lesion. CT images were instantly procured to evaluate the biopsy needle and pulmonary nodule's position at the desired depth. The procedure was continued only if the needle was at an appropriate site within or near the lesions, and the operator triggered the biopsy switch to trap the tissue specimens. Biopsy specimens were then preserved in $10 \%$ buffered 


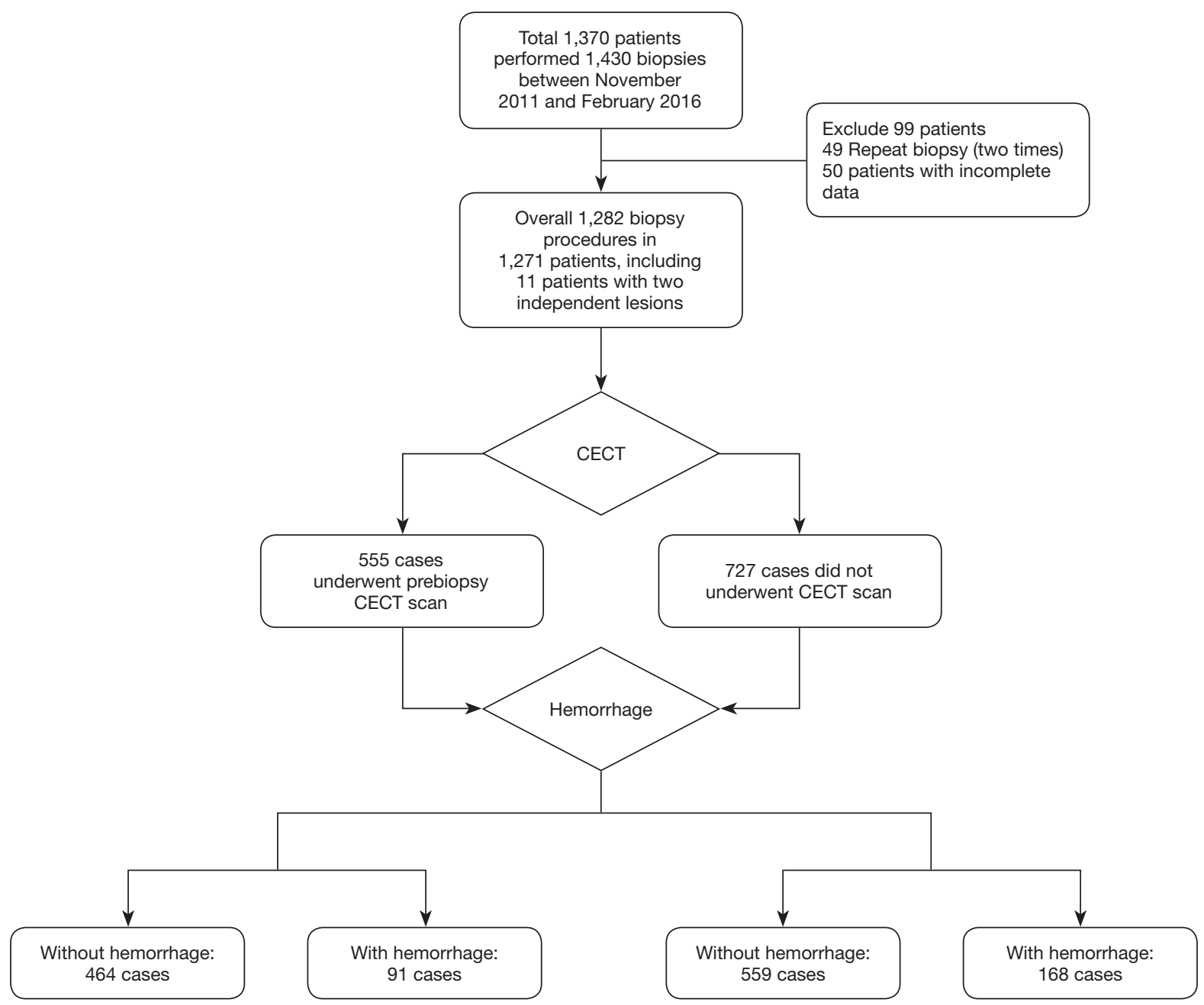

Figure 1 Flow diagram of patient inclusion and exclusion criteria.

formalin for pathological examination. After withdrawing the biopsy needle, a post-biopsy CT scan was immediately performed to assess possible complications, and the patient was required to stay in the supine position for at least $6 \mathrm{~h}$. A chest radiograph was generally performed the following day. All patients who underwent PTNB were inpatients, and if required after the biopsy, received oxygen therapy or hemostatic drugs, or underwent chest drainage or pulmonary artery embolization.

\section{Definitions}

The needle tracts' depths were calculated as the distance from the pleura to the center of the pulmonary lesions. Internal vessels were defined as vessels present at the edge and interior of the lesions, and others were considered peripheral vessels. Pulmonary hemorrhage was defined as any new haziness observed in the post-biopsy CT images. Bloody sputum was also considered a part of pulmonary hemorrhage. Mild hemorrhage was defined as hemorrhage that occurred in the pulmonary parenchyma or bloody sputum $(\leq 5 \mathrm{~mL})$, and severe hemorrhage was defined as hemoptysis complicating hemodynamic instability. The rest, exception of mild and severe hemorrhage, was defined as moderate hemorrhage.

\section{Statistical analysis}

All statistical analyses were performed using SPSS version 17 (SPSS, Chicago, IL, USA). Univariate analyses of categorical variables, such as sex and biopsy positions, were performed using $\chi^{2}$-test and Fisher's exact test, and 
continuous variables with skewed distributions were compared using the Mann-Whitney $U$-test. Multivariate logistic regression analyses of selected variables were performed to evaluate the effect of pre-biopsy CECT on hemorrhage rates and to determine the risk factors associated with hemorrhage. To demonstrate the effect of pre-biopsy CECT on hemorrhage rates, PSM was conducted by MatchIt package in $\mathrm{R}$ with nearest-neighbor 1:1, 2:1, and 3:1 matching. Differences and associations were considered significant when $\mathrm{P}<0.05$.

\section{Results}

\section{Patient characteristics}

A total of 1,271 patients (1,282 biopsies) were enrolled in the present study (854 males and 417 females), with a mean age of $59.0 \pm 2.72$ years. Of all 1,282 biopsies, the majority $(65.1 \%, \mathrm{n}=834)$ had a $>3 \mathrm{~cm}$ lesion size. Malignant and benign lesions accounted for $64 \%(n=820)$ and $29.7 \%$ $(\mathrm{n}=381)$, respectively. The most common lesion location was the upper lobe $(51.1 \%, \mathrm{n}=655)$, and the prone position was the main puncture position $(53.4 \%, \mathrm{n}=684)$. Also, 555 underwent CECT and 727 underwent non-contrast CT. Baseline characteristics, such as age, sex, smoking history, lesion size, and puncture position were not different between the CECT group and the non-contrast CT group $(\mathrm{P}>0.05)$ (Table 1).

\section{Association of CECT with the reduced incidence of post- biopsy bemorrbage}

Of the 1,282 PTNB, pulmonary hemorrhage occurred in $259(20.2 \%)$ biopsies. Hemorrhage rates in the CECT and the non-contrast CT were $16.4 \%$ (91/555) and $23.1 \%$ (168/721), respectively, which indicated that CECT before PTNB could reduce the incidence of biopsy-related hemorrhage $(\mathrm{P}=0.003)$. However, there was no statistical difference in hemorrhage grade (mild, moderate/severe) between the CECT and non-contrast CT groups $(\mathrm{P}=1.000)$. Of the 91 patients with hemorrhage in the CECT group, $95.6 \%(87 / 91)$ were classified as mild-grade hemorrhage, while $4.4 \%$ (4/91) as moderate/severe grade. In the noncontrast CT group, $95.2 \%(160 / 168)$ were classified as mild hemorrhage, and $4.8 \%(8 / 168)$ were classified as moderate/ severe hemorrhage (Table 2).

Also, vessel distribution observed by CECT, either in the peripheral $(\mathrm{P}=0.016)$ or internal $(\mathrm{P}=0.000)$ lesion, was associated with post-biopsy hemorrhage (Table 3).

\section{PSM analysis confirming the positive effects of CECT on reducing hemorrbage}

To reduce bias, PSM analysis was performed. In the CECT and the non-contrast CT groups, the following factors were matched by the MatchIt package in R, with nearestneighbor 1:1, 2:1, and 3:1 matching: sex, age, smoking history, lesion location, puncture position, size of the lesion, number of punctures, and depth of the needle tract (Table 4). According to 1:1 matching of hemorrhage and nonhemorrhage cases, the distribution of the propensity score is shown in Figure 2A. A significant negative relationship was found between pre-biopsy CECT and pulmonary hemorrhage $(\mathrm{P}=0.039)$. Figure $2 B, C$ shows the distribution of the propensity score from matching at a ratio of $1: 2$ $(\mathrm{P}=0.028)$ and $1: 3(\mathrm{P}=0.013)$, respectively.

\section{Univariate analysis of factors associated with hemorrhage}

We further analyzed factors that were assumed to be associated with post-biopsy hemorrhage; the results are summarized in Table 5. The data showed that females $(\mathrm{P}=0.039)$, patients with a lesion size $\leq 3 \mathrm{~cm}(\mathrm{P}=0.024)$, supine puncture position $(\mathrm{P}=0.008)$, repeated punctures $(\geq 2$, $\mathrm{P}=0.006)$, and depth of the needle tract $>4 \mathrm{~cm}(\mathrm{P}=0.012)$ were prone to hemorrhage. Hemorrhage was not found to be correlated with age, site of the lesion, and pathological results $(\mathrm{P}=0.160, \mathrm{P}=0.155$, and $\mathrm{P}=0.539$, respectively).

\section{Multivariate logistic regression analysis for risk factors of bemorrbage}

A further multivariate logistic regression model was established. We found that the incidence of post-biopsy hemorrhage was remarkably reduced in patients who underwent pre-biopsy CECT scans [odds ratio (OR): 0.671, 95\% confidence interval $(\mathrm{CI}): 0.499-0.902, \mathrm{P}=0.008]$. In addition, smaller lesion size (OR: 0.990, 95\% CI: $0.983-$ 0.997, $\mathrm{P}=0.005$ ), supine position during puncture (OR: 2.734. 95\% CI: $1.207-6.194, \mathrm{P}=0.016)$, increased number of punctures ( $\mathrm{n}=2$, OR: $1.546,95 \%$ CI: $1.065-2.244, \mathrm{P}=0.022$; $\mathrm{n}=3$, OR: $1.673,95 \%$ CI: $1.082-2.588, \mathrm{P}=0.021 ; \mathrm{n}=4$, OR: 8.746, 95\% CI: $2.891-26.456, \mathrm{P}<0.001)$, and increased depth of the needle tract (OR: 1.017, 95\% CI: 1.009-1.025, $\mathrm{P}=0.000)$ were all associated with upregulated risk of pulmonary hemorrhage (Table 6). 
Table 1 Patient demographics and lesion characteristics

\begin{tabular}{|c|c|c|c|c|}
\hline Characteristic & \multicolumn{2}{|c|}{ CECT $(n=555)$} & \multicolumn{2}{|c|}{ Non-contrast CT $(\mathrm{n}=727)$} \\
\hline \multicolumn{5}{|l|}{ Age (years) } \\
\hline$<65$ & 357 & 64.3 & 468 & 64.4 \\
\hline$\geq 65$ & 198 & 35.7 & 259 & 35.6 \\
\hline Male & 365 & 65.8 & 494 & 68.0 \\
\hline Female & 190 & 34.2 & 233 & 32.0 \\
\hline \multicolumn{5}{|c|}{ Smoking history (year package) } \\
\hline$\leq 20$ & 375 & 67.5 & 472 & 64.9 \\
\hline Supine & 229 & 41.3 & 293 & 40.3 \\
\hline Prone & 295 & 53.2 & 389 & 53.5 \\
\hline Lateral & 31 & 5.5 & 45 & 6.2 \\
\hline \multicolumn{5}{|l|}{ Lesion location } \\
\hline Upper lobe & 268 & 48.3 & 387 & 53.2 \\
\hline Middle lobe & 43 & 7.7 & 31 & 4.3 \\
\hline Lower lobe & 244 & 44.0 & 309 & 42.5 \\
\hline \multicolumn{5}{|l|}{ Lesion size $(\mathrm{cm})$} \\
\hline Non-diagnostic & 33 & 6.0 & 48 & 6.6 \\
\hline
\end{tabular}

CECT, contrast-enhanced computed tomography; CT, computed tomography.

\section{Discussion}

To the best of our knowledge, the present study is the first large-sample comparative study to assess if prebiopsy CECT could reduce hemorrhage incidence. We retrospectively reviewed 1,282 PTNB and found that prebiopsy CECT was associated with a reduced incidence of biopsy-related hemorrhage compared with non-contrast CT (16.4\% vs. 23.1\%, P=0.003). CECT before PTNB could also reduce post-biopsy hemorrhage risk (OR: 0.671, 95\% CI: 0.499-0.902, $\mathrm{P}=0.008$ ).
CECT has been used to distinguish malignant lung lesions from benign lung lesions, evaluate indeterminate pulmonary nodules, and predict lung adenocarcinoma pathological grades (17-20). In the present study, the risk of pulmonary hemorrhage remarkably decreased when prebiopsy CECT was performed, demonstrating the advantages of pre-biopsy CECT in the assessment of vascular distribution. To minimize hemorrhage caused by needle biopsy, Wu et al. suggested that major central blood vessels and systemic arteries should be avoided (7). A previous report showed that blood vessels that are not visible on non- 
Table 2 Grade of hemorrhage complication after percutaneous transthoracic needle biopsy (PTNB) between CECT group and non-contrast CT group

\begin{tabular}{lccc}
\hline Grade & CECT $(n=555)$ & Non-contrast CT $(n=727)$ & P value \\
\hline No hemorrhage & 464 & 559 & $0.003^{* *}$ \\
Hemorrhage & 91 & 168 & 1.000 \\
Mild & 87 & 160 & 8 \\
Moderate/severe & 4 & &
\end{tabular}

Table 3 Hemorrhage and vessels distribution observed by contrast-enhanced computed tomography (CECT)

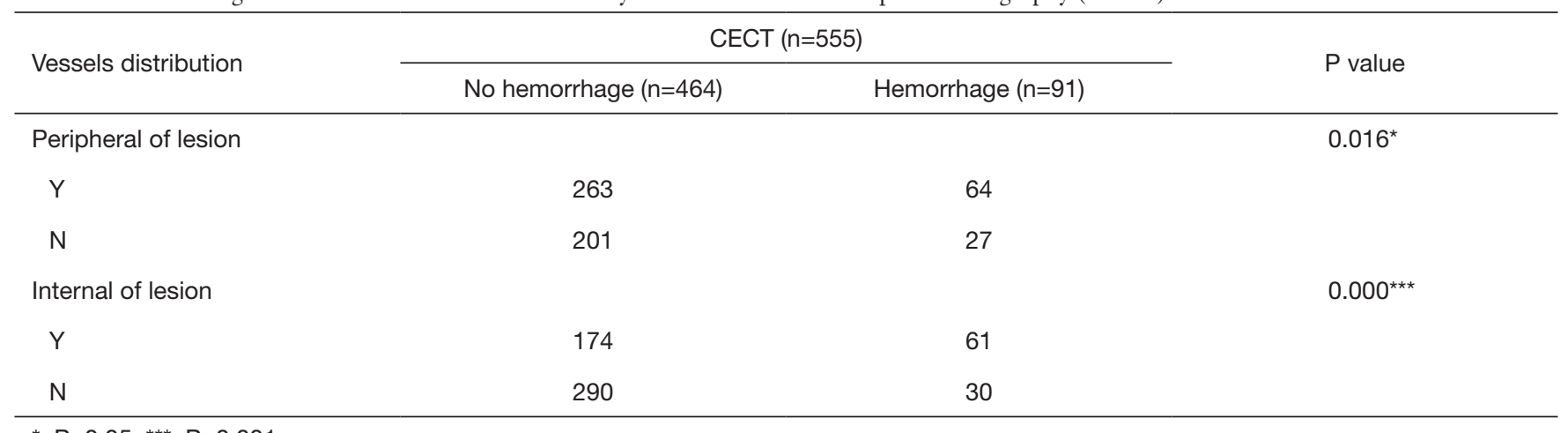

${ }^{*}, \mathrm{P}<0.05 ;{ }^{* * *}, \mathrm{P}<0.001$.

Table 4 The difference of hemorrhage incidence between non-contrast CT group and CECT group after matching hemorrhage and nonhemorrhage cases in variable proportions

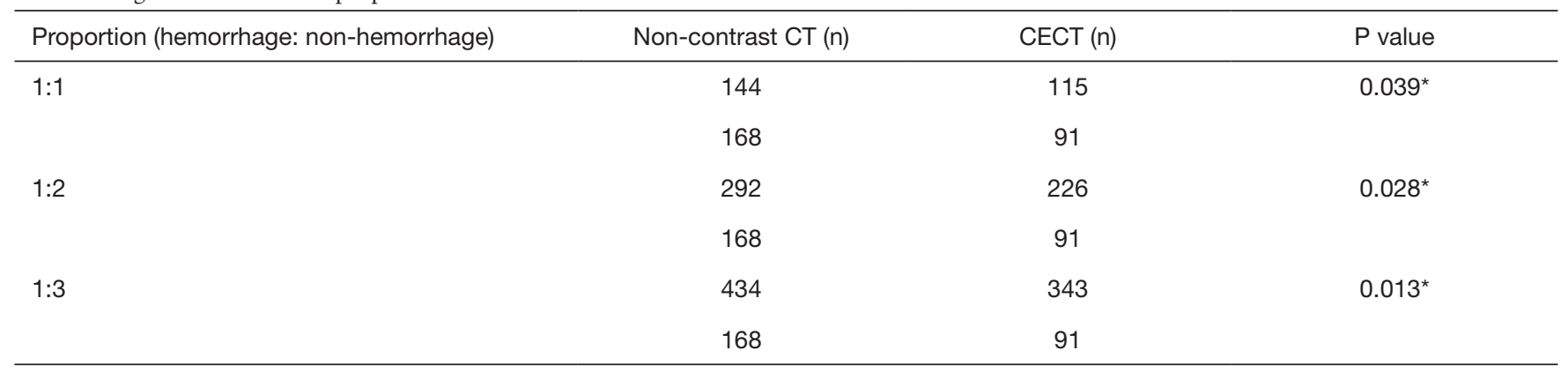

*, $\mathrm{P}<0.05$. CECT, contrast-enhanced computed tomography.

contrast CT images might be visible on CECT images (15). Nour-Eldin et al. showed that needle tracks across pulmonary blood vessels could increase hemorrhage risk (11). Similarly, the incidence of hemorrhage increased in the presence of abundant internal blood vessels or margins surrounded by blood vessels in the present study. The present study also confirmed that small blood vessels were significantly enhanced in arterial and venous phases on CECT images, facilitating more accurate identification of suitable puncture routes that avoid blood vessels.

Baadh et al. showed that using a track embolization technique with absorbable hemostat gelatin powder reduces hemorrhage (21). A limitation of this method was that the duration of the overall procedure, which was extended by approximately 1-2 $\mathrm{min}$, potentially increased the risk of pneumothorax. Khan et al. also showed that pneumothorax rates significantly increased with an increase in puncture time. Compared with track embolization techniques, pre- 

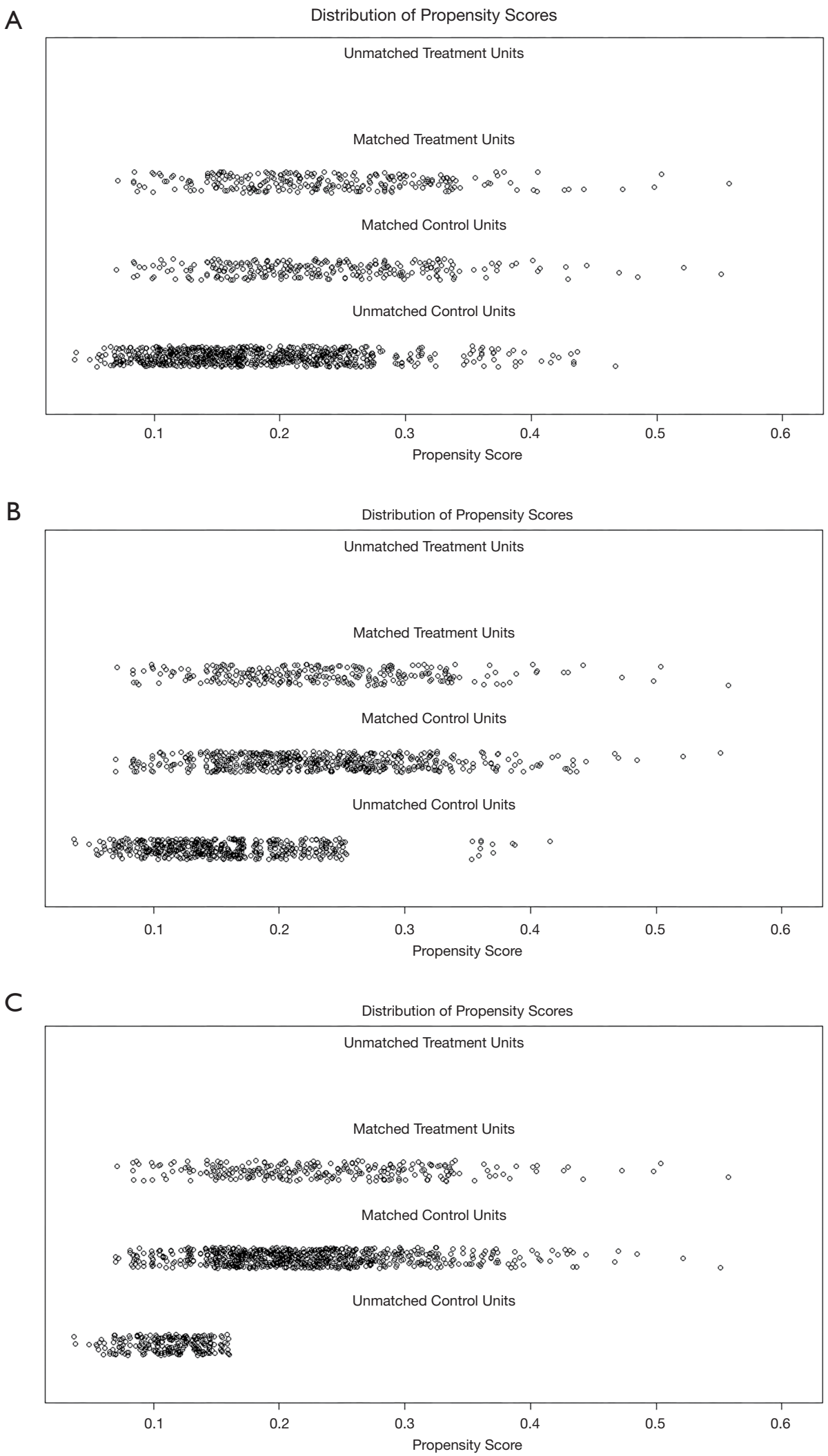

Figure 2 Propensity score distribution of hemorrhage and non-hemorrhage patients after matching at different ratios. (A) 1:1; (B) 1:2; (C) 1:3. 
Table 5 Univariate analysis of risk factors associated with hemorrhage after percutaneous transthoracic needle biopsy (PTNB)

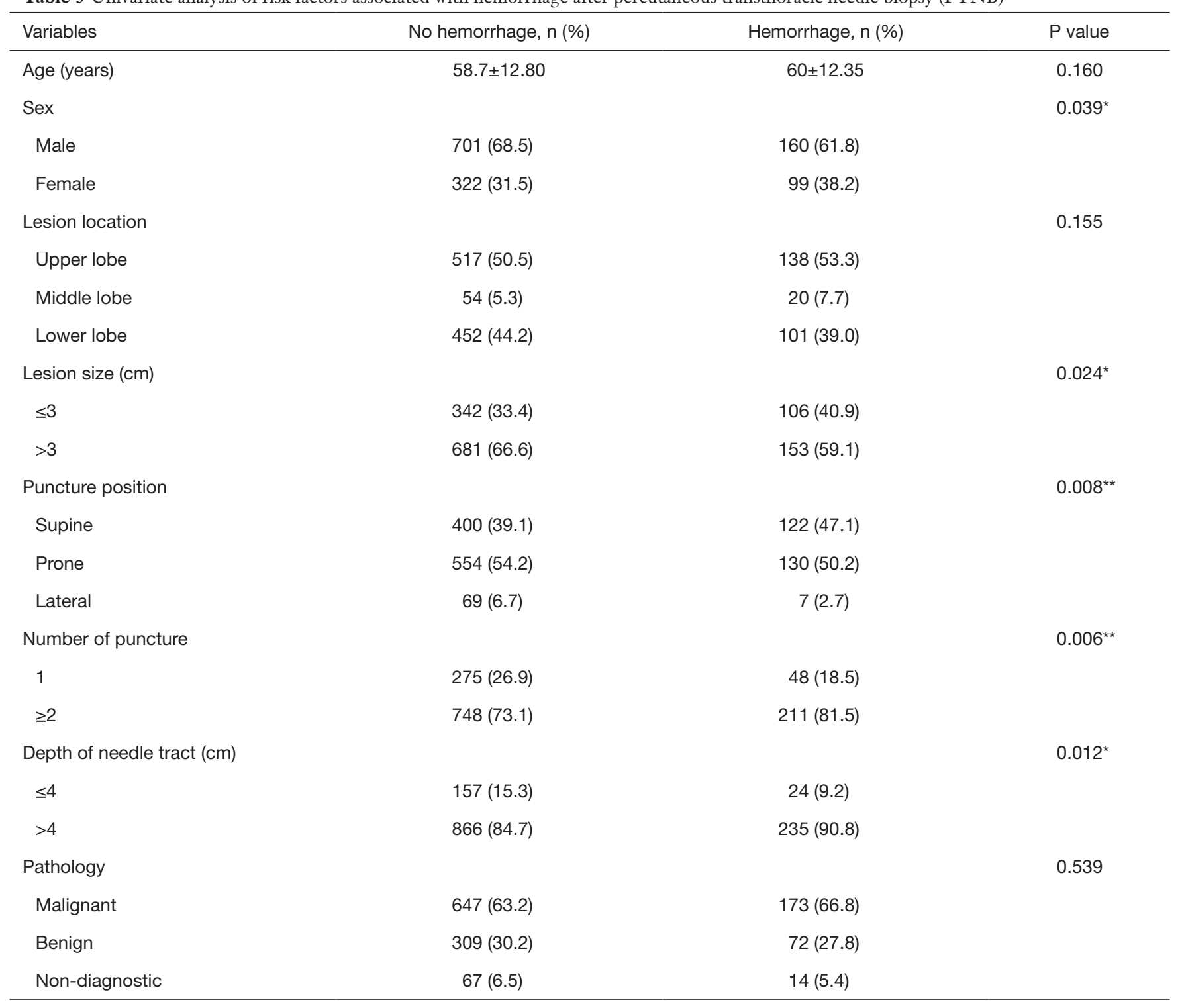

${ }^{*}, \mathrm{P}<0.05 ;{ }^{* *}, \mathrm{P}<0.01$.

biopsy CECT does not potentially increase the incidence of complications associated with puncture. The risks associated with the administration of intravenous injection iodine-based contrast agents, such as impaired renal function, contrast extravasation, and allergic reactions, were low if a full medical history and a history of allergies were provided. Therefore, we recommend using this convenient and safe technique before the biopsy to reduce pulmonary hemorrhage, which is the second most prevalent complication of PTNB.

As reported in previous studies, pulmonary hemorrhage is affected by factors, such as sex, site of the lesion, size of the lesion, and depth of the needle tract (22-24). Our PSM analysis excluded the effects of confounding factors and confirmed that pre-biopsy CECT reduced PTNBrelated hemorrhage. Significant differences in hemorrhage ratios between the pre-biopsy CECT and the non-CECT groups were observed in 1:1, 1:2, and 1:3 matching analyses, providing further evidence pre-biopsy CECT reduces the hemorrhage rate in patients undergoing PTNB. Previous research assessing risk factors of hemorrhage complicating needle biopsy did not involve methods, such as PSM, to 
Table 6 Multivariate logistic regression analysis for risk factors of hemorrhage

\begin{tabular}{lcc}
\hline Variables & OR $(95 \% \mathrm{Cl})$ & P value \\
\hline CECT & $0.671(0.499-0.902)$ & $0.008^{\star \star}$ \\
Y & Reference & \\
$\mathrm{N}$ & $0.990(0.983-0.997)$ & $0.005^{\star *}$ \\
Lesion size $(\mathrm{cm})$ & & \\
Puncture position & $2.734(1.207-6.194)$ & $0.016^{\star}$ \\
Supine & $2.107(0.932-4.764)$ & 0.073 \\
Prone & Reference & \\
Lateral & Reference & \\
Number of puncture & $1.546(1.065-2.244)$ & $0.022^{\star}$ \\
1 & $1.673(1.082-2.588)$ & $0.021^{\star}$ \\
2 & $8.746(2.891-26.456)$ & $0.000^{\star \star *}$ \\
3 & $1.017(1.009-1.025)$ & $0.000^{\star \star *}$ \\
4 & &
\end{tabular}

${ }^{*}, \mathrm{P}<0.05 ;{ }^{* *}, \mathrm{P}<0.01$; ${ }^{* *}, \mathrm{P}<0.001$. OR, odd ratio; $\mathrm{Cl}$, confidence interval; CECT, contrast-enhanced computed tomography.

control the influence of confounding factors. Therefore, to our knowledge, the present study is the first largesample study to convincingly demonstrate that pre-biopsy CECT scans can be used to reduce the risk of pulmonary hemorrhage complications in patients undergoing PTNB.

Although our research has significant clinical value, it has several limitations. Biopsy procedures were conducted by experienced Operators, the differences between operators were not considered, which might affect the incidence of complication.

Second, it was a retrospective and single-center study, which could introduce unavoidable bias. For example, at the early stage, the role of CECT may not be recognized and the proportion of performing CECT was relatively low compared to the later stage, which might introduce distribution bias. Our findings should be validated in future studies with larger cohorts from multi-centers.

\section{Conclusions}

The present study confirmed that pre-biopsy CECT was an effective tool to prevent pulmonary hemorrhage. Further studies are required to establish CECT as a routine pre- biopsy procedure in patients scheduled to undergo PTNB.

\section{Acknowledgments}

Funding: None.

\section{Footnote}

Reporting Checklist: The authors have completed the STROBE reporting checklist. Available at: http://dx.doi. org/10.21037/atm-20-4384

Data Sharing Statement: Available at http://dx.doi. org/10.21037/atm-20-4384

Conflicts of Interest: All authors have completed the ICMJE uniform disclosure form (available at http://dx.doi. org/10.21037/atm-20-4384). The authors have no conflicts of interest to declare.

Ethical Statement: The authors are accountable for all aspects of the work in ensuring that questions related to the accuracy or integrity of any part of the work are appropriately investigated and resolved. This study was conducted in accordance with the Declaration of Helsinki (as revised in 2013) and approved by the institutional review board of Jinling Hospital (No. 2017NZHX-022). Informed consent from individuals for this retrospective analysis was waived.

Open Access Statement: This is an Open Access article distributed in accordance with the Creative Commons Attribution-NonCommercial-NoDerivs 4.0 International License (CC BY-NC-ND 4.0), which permits the noncommercial replication and distribution of the article with the strict proviso that no changes or edits are made and the original work is properly cited (including links to both the formal publication through the relevant DOI and the license). See: https://creativecommons.org/licenses/by-nc-nd/4.0/.

\section{References}

1. Tam AL, Kim ES, Lee JJ, et al. Feasibility of image-guided transthoracic core-needle biopsy in the BATTLE lung trial. J Thorac Oncol 2013;8:436-42.

2. Cheung YC, Chang JW, Hsieh JJ, et al. Adequacy and complications of computed tomography-guided core needle biopsy on non-small cell lung cancers for epidermal 
growth factor receptor mutations demonstration: 18-gauge or 20-gauge biopsy needle. Lung Cancer 2010;67:166-9.

3. Heerink WJ, de Bock GH, de Jonge GJ, et al. Complication rates of CT-guided transthoracic lung biopsy: meta-analysis. Eur Radiol 2017;27:138-48.

4. Ferretti GR, Busser B, de Fraipont F, et al. Adequacy of CT-guided biopsies with histomolecular subtyping of pulmonary adenocarcinomas: influence of ATS/ERS/ IASLC guidelines. Lung Cancer 2013;82:69-75.

5. Nakatani M, Tanigawa N, Kariya S, et al. Analysis of factors influencing accuracy and complications in CTguided lung biopsy. Minim Invasive Ther Allied Technol 2012;21:415-22.

6. Heck SL, Blom P, Berstad A. Accuracy and complications in computed tomography fluoroscopy-guided needle biopsies of lung masses. Eur Radiol 2006;16:1387-92.

7. Wu CC, Maher MM, Shepard JA. Complications of CT-guided percutaneous needle biopsy of the chest: prevention and management. AJR. AJR Am J Roentgenol 2011;196:W678-82.

8. Wang Y, Li W, He X, et al. Computed tomography-guided core needle biopsy of lung lesions: Diagnostic yield and correlation between factors and complications. Oncol Lett 2014;7:288-94.

9. Yildirim E, Kirbas I, Harman A, et al. CT-guided cutting needle lung biopsy using modified coaxial technique: factors effecting risk of complications. Eur J Radiol 2009;70:57-60.

10. Billich C, Muche R, Brenner G, et al. CT-guided lung biopsy: incidence of pneumothorax after instillation of $\mathrm{NaCl}$ into the biopsy track. Eur Radiol 2008;18:1146-52.

11. Nour-Eldin NE, Alsubhi M, Naguib NN, et al. Risk factor analysis of pulmonary hemorrhage complicating CTguided lung biopsy in coaxial and non-coaxial core biopsy techniques in 650 patients. Eur J Radiol 2014;83:1945-52.

12. Wang Y, Jiang F, Tan X, et al. CT-guided percutaneous transthoracic needle biopsy for paramediastinal and nonparamediastinal lung lesions: Diagnostic yield and complications in 1484 patients. Medicine 2016;95:e4460.

13. Tomiyama N, Yasuhara Y, Nakajima Y, et al. CTguided needle biopsy of lung lesions: a survey of severe complication based on 9783 biopsies in Japan. Eur J Radiol 2006;59:60-4.

14. Manhire A, Charig M, Clelland C, et al. Guidelines for radiologically guided lung biopsy. Thorax 2003;58:920-36.

15. Oh JK, Han DH, Ko JM. The internal mammary vessels above and below the first rib on multidetector CT: implications for anatomical feasibility of lung biopsy via anterior approach. Diagn Interv Radiol 2011;17:223-8.

16. Yeow KM, Su IH, Pan KT, et al. Risk factors of pneumothorax and bleeding: multivariate analysis of 660 CT-guided coaxial cutting needle lung biopsies. Chest 2004;126:748-54.

17. Dabrowska M, Krenke R, Korczynski P, et al. Diagnostic accuracy of contrast-enhanced computed tomography and positron emission tomography with $18-\mathrm{FDG}$ in identifying malignant solitary pulmonary nodules. Medicine 2015;94:e666.

18. Dabrowska M, Zukowska M, Krenke R, et al. Simplified method of dynamic contrast-enhanced computed tomography in the evaluation of indeterminate pulmonary nodules. Respiration 2010;79:91-6.

19. Pinstein ML, Scott RL, Salazar J. Avoidance of negative percutaneous lung biopsy using contrast-enhanced CT. AJR Am J Roentgenol 1983;140:265-7.

20. Liu Y, Liu S, Qu F, et al. Tumor heterogeneity assessed by texture analysis on contrast-enhanced CT in lung adenocarcinoma: association with pathologic grade. Oncotarget 2017;8:53664-74.

21. Baadh AS, Hoffmann JC, Fadl A, et al. Utilization of the track embolization technique to improve the safety of percutaneous lung biopsy and/or fiducial marker placement. Clin Imaging 2016;40:1023-8.

22. Khan MF, Straub R, Moghaddam SR, et al. Variables affecting the risk of pneumothorax and intrapulmonal hemorrhage in CT-guided transthoracic biopsy. Eur Radiol 2008;18:1356-63.

23. Wei YH, Zhou FX, Li Y, et al. Extrapleural locating method in computed tomography-guided needle biopsies of 1,106 lung lesions. Exp Ther Med 2015;10:1707-19.

24. Tai R, Dunne RM, Trotman-Dickenson B, et al. Frequency and Severity of Pulmonary Hemorrhage in Patients Undergoing Percutaneous CT-guided Transthoracic Lung Biopsy: Single-Institution Experience of 1175 Cases. Radiology 2016;279:287-96.

Cite this article as: $\mathrm{Hu} \mathrm{H}, \mathrm{Li} \mathrm{C}, \mathrm{Lv} \mathrm{T}, \mathrm{Li} \mathrm{H}, \mathrm{Hu}$ Y, Shen Q, Mino-Kenudson M, Bertolaccini L, Rocco G, Zarogoulidis P, Zhang F, Lin D, Liu H, Song Y; written on behalf of AME Pulmonary Disease Collaborative Group. Contrast-enhanced computed tomography prior to percutaneous transthoracic needle biopsy reduces the incidence of hemorrhage. Ann Transl Med 2021;9(4):288. doi: 10.21037/atm-20-4384 